\title{
Dark Matter Interacts with Electromagnetic Waves
}

\author{
Koshun Suto \\ Chudai-Ji Temple, Isesaki, Japan \\ Email: koshun_suto129@mbr.nifty.com
}

How to cite this paper: Suto, K. (2021) Dark Matter Interacts with Electromagnetic Waves. Journal of High Energy Physics, Gravitation and Cosmology, 7, 1298-1305. https://doi.org/10.4236/jhepgc.2021.74079

Received: July 15, 2021

Accepted: September 5, 2021

Published: September 8, 2021

Copyright (C 2021 by author(s) and Scientific Research Publishing Inc. This work is licensed under the Creative Commons Attribution International License (CC BY 4.0).

http://creativecommons.org/licenses/by/4.0/

\begin{abstract}
The large-scale structure of DM cannot be directly seen, but it is believed it can be inferred from the distribution of visible galaxies formed from ordinary matter. Bright visible galaxies that emit the Lyman $\alpha(\operatorname{Ly} \alpha)$ emission line of the hydrogen atom (Ly $\alpha$ galaxies) are used to observe the large-scale structure of distant space. However, recently Momose et al. have reported cases where the large-scale structures of DM indicated by Ly $\alpha$ galaxies and other galaxies fail to match. This raises the possibility that Ly $\alpha$ galaxies may not correctly indicate the large-scale structure of DM. In the currently accepted cold DM model, DM and neutral hydrogen gas are thought to interact only through the mutual effects of gravity. However, according to Suto, DM and ordinary matter are like two sides of the same coin. By giving and receiving approximately $2 m_{\mathrm{e}} \mathrm{c}^{2}(1.022 \mathrm{MeV})$, it is possible to mutually convert between the two. If, in future observations of the density distribution of interstellar gas using Ly $\alpha$ emission lines, unexpected data is obtained that cannot be explained based only on absorption by neutral hydrogen gas, then the author believes the problem can be solved with Suto's DM model.
\end{abstract}

\section{Keywords}

Dark Matter, Dark Hydrogen Atom, Lyman $\alpha$ Emission, Einstein's

Energy-Momentum Relationship, Suto's Energy-Momentum Relationship

\section{Introduction}

It is believed that the largest structures in the universe are large-scale structures made of dark matter (DM), a material which cannot be directly observed. This DM forms a large-scale web-like structure. It is thought that galaxies, like the Milky Way where we live, are formed within the filaments of this large-scale structure. 
It is thought that his large-scale structure was formed due to slight quantum fluctuations when the universe was born. It is believed that those fluctuations later caused irregularities in the density of DM. Then those irregularities gradually expanded due to the effects of gravity. It is believed that DM only interacts with ordinary matter through gravity, and thus cannot be observed using any type of electromagnetic wave.

There is also the following very similar explanation. According to the currently favored model of the formation of the universe (the cold DM model), it is believed that roughly 10 to 11.5 billion years ago large amounts of hydrogen gas expanded out like a web, and astronomical objects such as the fixed stars and galaxies came into being in the mesh of that web.

The large amounts of hydrogen gas that float in space are attracted to each other due to their mutual gravity (universal gravitation) and form into more dense clouds. As the density of hydrogen gas increases, the surrounding hydrogen gas is drawn in, so the clouds grow into larger astronomical objects.

Recently, Umehata et al. observed, for the first time in the world, some of the hydrogen gas distributed in a web-like form, which had previously been predicted but never actually observed [1].

According to the above explanation, the large-scale structure of the universe is formed of DM and hydrogen gas. The distribution of hydrogen gas is similar to the density distribution of DM.

The large-scale structure of DM cannot be directly seen, but it is believed it can be inferred from the distribution of visible galaxies formed from ordinary matter.

Bright visible galaxies that emit the $\operatorname{Lyman} \alpha(\operatorname{Ly} \alpha)$ emission line of the hydrogen atom (Ly $\alpha$ galaxies) are used to observe the large-scale structure of distant space.

However, recently Momose et al. have reported cases where the large-scale structures of DM indicated by Ly $a$ galaxies and other galaxies fail to match. This raises the possibility that Ly $\alpha$ galaxies may not correctly indicate the large-scale structure of DM [2].

Momose et al. have worked to elucidate this problem by using observation data on intergalactic neutral hydrogen gas. As samples, they selected three types of galaxies from among galaxies at a distance of approximately 10 million light-years. The three types of galaxies are as follows:

1) Ly $\alpha$ galaxies

2) Continuous light galaxies which emit light uniformly at all wavelengths

3) Visible emission line galaxies which shine with the green emission line emitted by oxygen

Momose et al. divided into the near side of each galaxy (region close to the earth) and far side of each galaxy (region beyond the galaxy) and observed the intergalactic gas density distribution. For continuous light galaxies and visible emission line galaxies, the density distributions of interstellar gas on the near 
and far sides of the galaxies were almost the same. However, in the case of Ly $\alpha$ galaxies, it was found that the intergalactic gas on the near side of the galaxy was lower in density than gas on the far side. These observations imply that the Ly $\alpha$ galaxies we can observe are on the near side of the high-density region of neutral hydrogen gas.

Momose et al. explained that the reason why this situation occurred is because Ly $\alpha$ galaxies are absorbed and scattered by neutral hydrogen gas on the near side of the galaxy.

It is believed that DM does not ordinarily absorb or emit light.

However, Suto proposed a model of DM capable of absorbing Ly $\alpha$ emission lines [3]. In the opinion of Suto, neutral hydrogen gas is not the only thing that can absorb Ly $\alpha$ emission lines.

\section{Overview of Model of DM Capable of Absorbing Ly $\alpha$ Emission Lines}

In the hydrogen atom, there are energy levels far lower than the ordinary known energy levels. Electrons at these energy levels have negative mass. Suto uses the name "dark hydrogen atom" (DHA) for matter formed from these electrons with negative mass and protons with positive mass. An overview of the results derived by Suto is given below [4].

The energy-momentum relationship in the special theory of relativity (STR) holds in an isolated system in free space. Here, if $m_{0} c^{2}$ is the rest mass energy and $m c^{2}$ is the relativistic energy, the relationship can be written as follows.

$$
\left(m_{0} c^{2}\right)^{2}+p^{2} c^{2}=\left(m c^{2}\right)^{2} .
$$

The following equation holds due to Formula (1).

$$
\left(m c^{2}\right)^{2}=m_{0}^{2} c^{4}+\left(m^{2} c^{4}-m_{0}^{2} c^{4}\right)=\left(m_{0} c^{2}\right)^{2}+\left(m+m_{0}\right)\left(m c^{2}-m_{0} c^{2}\right) c^{2} .
$$

Using this, Formula (1) becomes as follows.

$$
\left(m_{0} c^{2}\right)^{2}+\left(m+m_{0}\right)\left(m c^{2}-m_{0} c^{2}\right) c^{2}=\left(m c^{2}\right)^{2} .
$$

Incidentally, Sommerfeld and Einstein defined relativistic kinetic energy as follows [5].

$$
K_{\mathrm{re}}=m c^{2}-m_{0} c^{2} .
$$

Since this equation and Formula (1) are equal, the following relationship must hold when Formula (4) is taken into account.

$$
p^{2}=\left(m_{0}+m\right)\left(m c^{2}-m_{0} c^{2}\right)=\left(m_{0}+m\right) K_{\mathrm{re}} .
$$

The following formula is obtained from this.

$$
K_{\mathrm{re}}=\frac{p^{2}}{m+m_{0}} .
$$

Next, the relativistic kinetic energy of an electron in a hydrogen atom is defined as follows by referring to Formula (6). 


$$
K_{\text {re }, n}=\frac{p_{\text {re }, n}^{2}}{m_{\mathrm{e}}+m_{n}}, \quad p_{\text {re }, n}=m_{n} v_{n} .
$$

Here, $m_{n}$ is the relativistic mass of the electron. Also, $p_{\mathrm{re}, n}$ indicates the relativistic momentum of the electron.

Incidentally, the energy of an electron at rest in an isolated system in free space is $m_{\mathrm{e}} c^{2}$. Here, we consider the case where this electron is drawn in by the electrical attraction of the proton, and forms a hydrogen atom. At this time, the electron emits a photon to the outside. Therefore, the relativistic energy of an electron in a hydrogen atom $m_{n} c^{2}$ becomes smaller than the rest mass energy $m_{\mathrm{e}} c^{2}$. That is,

$$
m_{n} c^{2}<m_{\mathrm{e}} c^{2}
$$

The behavior of an electron inside an atom, where there is potential energy, cannot be described with the relationship of Einstein (1). Caution is necessary because it is completely overlooked in Formula (8).

Now, referring to Formula (4), it is natural to define the relativistic kinetic energy of an electron in a hydrogen atom as follows.

$$
K_{\mathrm{re}, n}=-E_{\mathrm{re}, n}=m_{\mathrm{e}} c^{2}-m_{n} c^{2} .
$$

This paper defines $E_{\mathrm{re}, n}$ as the relativistic energy levels of the hydrogen atom derived at the level of classical quantum theory. (The quantum number used here is just the principal quantum number. Therefore, $E_{\mathrm{re}, n}$ is not a formula which predicts all the relativistic energy levels of the hydrogen atom.)

However, the term "relativistic" used here does not mean based on the special theory of relativity (STR). It means that the expression takes into account the fact that the mass of the electron varies due to velocity. According to the STR, the electron's mass increases when its velocity increases. However, inside the hydrogen atom, the mass of the electron decreases when the velocity of the electron increases. Attention must be paid to the fact that, inside the hydrogen atom, the relativistic mass of the electron $m_{n}$ is smaller than the rest mass $m_{\mathrm{e}}$.

In this way, two formulas have been obtained for the relativistic kinetic energy of the electron in a hydrogen atom (Formulas (7) and (9)).

Incidentally, the following equation can be derived from Formulas (7) and (9).

$$
\frac{p_{\mathrm{re}, n}^{2}}{m_{\mathrm{e}}+m_{n}}=m_{\mathrm{e}} c^{2}-m_{n} c^{2} .
$$

Rearranging this, the following relationship can be derived.

$$
\left(m_{n} c^{2}\right)^{2}+p_{\mathrm{re}, n}^{2} c^{2}=\left(m_{\mathrm{e}} c^{2}\right)^{2} .
$$

Formula (11) is the energy-momentum relationship applicable to the electron in a hydrogen atom. (Suto calls this "Suto's energy-momentum relationship") [6].

By solving this relationship (11), it is possible to derive the relativistic energy levels $E_{\mathrm{re}, n}^{+}$of the hydrogen atom as follows [7]. 


$$
E_{\mathrm{re}, n}^{+}=m_{n} c^{2}-m_{\mathrm{e}} c^{2}=m_{\mathrm{e}} c^{2}\left[\frac{n}{\left(n^{2}+\alpha^{2}\right)^{1 / 2}}-1\right] .
$$

Suto has shown that these energy levels match the energy levels $E_{\mathrm{BO}, n}$ derived approximately by Bohr [8]. That is,

$$
E_{\mathrm{re}, n}^{+} \approx E_{\mathrm{BO}, n}=-\frac{\alpha^{2}}{2 n^{2}} m_{\mathrm{e}} c^{2} .
$$

Here, $\alpha$ is the following fine-structure constant.

$$
\alpha=\frac{e^{2}}{4 \pi \varepsilon_{0} \hbar c}=7.2973525693 \times 10^{-3} .
$$

There is a reason why relativistic energy was expressed as $E_{\mathrm{re}, n}^{+}$in Formula (12). Formula (11) is a quadratic equation, and thus there is also a negative solution. This is similar to the fact that there are positive and negative solutions to Einstein's relationship (1).

Expressing the negative solution here as $E_{\mathrm{re}, n}^{-}$,

$$
E_{\mathrm{re}, n}^{-}=-2 m_{\mathrm{e}} c^{2}+E_{\mathrm{re}, n}^{+} \approx-2 m_{\mathrm{e}} c^{2}+\frac{\alpha^{2}}{2 n^{2}} m_{\mathrm{e}} c^{2} .
$$

The following Figure 1 illustrates the relationship between $E_{\mathrm{re}, n}^{+}$and $E_{\mathrm{re}, n}^{-}$.

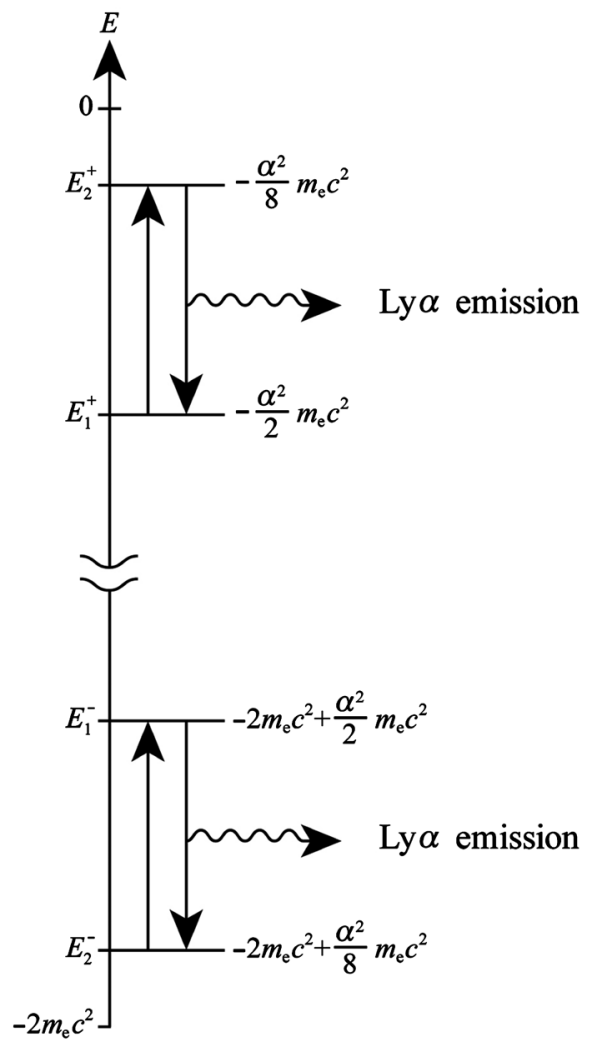

Figure 1. Energy levels of ordinary hydrogen atoms $E_{\mathrm{re}, n}^{+}$and energy levels of dark hydrogen atoms (DHA) $E_{\mathrm{re}, n}^{-}$. Ly $\alpha$ emission lines are absorbed in the transition from $n=1$ to 2 in a hydrogen atom, and in the transition from $n=2$ to 1 in a DHA. 
Energy is the same for photons emitted from ordinary hydrogen atoms and from DHA. Therefore, even if a photon is emitted from a DHA, it is impossible to discriminate between that photon and a photon emitted from an ordinary hydrogen atom.

Similarly, even if a Ly $\alpha$ emission line is absorbed, it cannot be determined whether it was absorbed by a hydrogen atom or a DHA.

This situation appears to be why DM is believed to not emit or absorb light.

Next, if the electron orbital radii corresponding to the energy levels in Formulas (13) and (15) are taken to be, respectively, $r_{n}^{+}$and $r_{n}^{-}$,

$$
\begin{aligned}
& r_{n}^{+}=\frac{r_{\mathrm{e}}}{2} \frac{\left(n^{2}+\alpha^{2}\right)^{1 / 2}}{\left(n^{2}+\alpha^{2}\right)^{1 / 2}-n} . \\
& r_{n}^{-}=\frac{r_{\mathrm{e}}}{2} \frac{\left(n^{2}+\alpha^{2}\right)^{1 / 2}}{\left(n^{2}+\alpha^{2}\right)^{1 / 2}+n} .
\end{aligned}
$$

Also, Formulas (16) and (17) can be written as follows.

$$
\begin{aligned}
& r_{n}^{+}=\frac{r_{\mathrm{e}}}{2}\left[1+\frac{n}{\left(n^{2}+\alpha^{2}\right)^{1 / 2}-n}\right] . \\
& r_{n}^{-}=\frac{r_{\mathrm{e}}}{2}\left[1-\frac{n}{\left(n^{2}+\alpha^{2}\right)^{1 / 2}+n}\right] .
\end{aligned}
$$

The next compares the orbital radii of an electron in a hydrogen atom $r_{n}^{+}$ and the orbital radii of an electron with a negative mass $r_{n}^{-}$.

The following ratio is obtained from Formulas (16) and (17).

$$
\frac{r_{n}^{-}}{r_{n}^{+}}=\frac{\left(n^{2}+\alpha^{2}\right)^{1 / 2}-n}{\left(n^{2}+\alpha^{2}\right)^{1 / 2}+n} \text {. }
$$

Here, if we set $n=1$,

$$
\frac{r_{1}^{-}}{r_{1}^{+}}=\frac{\left(1+\alpha^{2}\right)^{1 / 2}-1}{\left(1+\alpha^{2}\right)^{1 / 2}+1}=1.3312484168 \times 10^{-5} \approx \frac{1}{75120}
$$

Suto pointed out that an electron with negative mass forming DHA exists near the atom's nucleus (proton) [9] [10]. A single DHA has about the same mass as an ordinary hydrogen atom. However, a DHA is far smaller than an ordinary hydrogen atom, and thus dark hydrogen can attain high density. Therefore, dark matter can be an important source of gravity.

\section{Conclusions}

In the DM model proposed by Suto, the DHA constituting some of DM can emit and absorb Ly $\alpha$ emission lines. Therefore, in the observations of Momose et al., there is a possibility that some of the Ly $\alpha$ emission lines thought to have been 
absorbed by neutral hydrogen gas were absorbed by DHA.

The DM model proposed by Suto has the following features.

1) Just like an ordinary hydrogen atom, a DHA can emit and absorb Ly $\alpha$ emission lines.

The Ly $\alpha$ emission line is absorbed in the transition from $n=1$ to 2 in the case of a hydrogen atom, and in the transition from $n=2$ to 1 in the case of a DHA.

2) The electrons composing an ordinary hydrogen atom and a DHA can become a DHA and hydrogen atom by giving and receiving a $\gamma$-ray of energy approximately $2 m_{\mathrm{e}} c^{2} \quad(1.022 \mathrm{MeV})$.

In the currently accepted cold DM model, DM and neutral hydrogen gas are thought to interact only through the mutual effects of gravity. However, according to Suto, DM and ordinary matter are like two sides of the same coin. By giving and receiving approximately $2 m_{\mathrm{e}} c^{2}(1.022 \mathrm{MeV})$, it is possible to mutually convert between the two.

If, in future observations of the density distribution of interstellar gas using Ly $\alpha$ emission lines, unexpected data is obtained that cannot be explained based only on absorption by neutral hydrogen gas, then the author believes the problem can be solved with Suto's DM model.

However, the author addresses the following minor, but important issues. The DM problem may be solved also through the extended theories of gravity [11].

\section{Acknowledgments}

I would like to express my thanks to the staff at ACN Translation Services for their translation assistance. Also, I wish to express my gratitude to Mr. H. Shimada for drawing figures.

\section{Conflicts of Interest}

The author declares no conflicts of interest regarding the publication of this paper.

\section{References}

[1] Umehara, H., et al. (2019) Gas Filaments of the Cosmic Web Located Around Active Galaxies in a Protocluster. Science, 366, 97-100. https://doi.org/10.1126/science.aaw5949

[2] Momose, R., et al. (2021) Catch Me If You Can: Biased Distribution of Ly $\alpha$-Emitting Galaxies According to the Viewing Direction. The Astrophysical Journal Letters, 912, 1-8. https://doi.org/10.3847/2041-8213/abf04c

[3] Suto, K. (2014) Previously Unknown Ultra-Low Energy Level of the Hydrogen Atom Whose Existence Can Be Predicted. Applied Physics Research, 6, 64-73. https://doi.org/10.5539/apr.v6n6p64

[4] Suto, K. (2017) Presentation of Dark Matter Candidates. Applied Physics Research, 9, 70-76. https://doi.org/10.5539/apr.v9n1p70

[5] Sommerfeld, A. (1923) Atomic Structure and Spectral Lines. Methuen \& Co. Ltd., London, 528. 
[6] Suto, K. (2021) The Quantum Condition That Should Have Been Assumed by Bohr When Deriving the Energy Levels of a Hydrogen Atom. Journal of Applied Mathematics and Physics, 9, 1230-1244. https://doi.org/10.4236/jamp.2021.96084

[7] Suto, K. (2019) The Relationship Enfolded in Bohr's Quantum Condition and a Previously Unknow Formula for Kinetic Energy. Applied Physics Research, 11, 19-34. https://doi.org/10.5539/apr.v11n1p19

[8] Suto, K. (2020) Dark Matter and the Energy-Momentum Relationship in a Hydrogen Atom. Journal of High Energy Physics, Gravitation and Cosmology, 6, 52-61. https://doi.org/10.4236/jhepgc.2020.61007

[9] Suto, K. (2017) Region of Dark Matter Present in the Hydrogen Atom. Journal of Physical Mathematics, 8, 1000252.

[10] Suto, K. (2020) The Prediction of Negative Energy Specific to the Electron. Journal of Modern Physics, 11, 712-724. https://doi.org/10.4236/jmp.2020.115046

[11] Corda, C, (2009) Interferometric Detection of Gravitational Waves: The Definitive Test for General Relativity. International Journal of Modern Physics D, 18, 2275-2282. https://doi.org/10.1142/S0218271809015904 